\title{
Hemmt Metformin das Mammakarzinom?
}

Postmenopausale Diabetikerinnen, die Metformin einnehmen, erkranken einer amerikanischen Studie zufolge seltener an Brustkrebs als Diabetikerinnen unter einer anderen Therapie oder Frauen ohne Diabetes.

$\mathrm{n}$ großen populationsbasierten Studien wurde deutlich, dass Diabetikerinnen im Vergleich zur Normalbevölkerung ein erhöhtes Brustkrebsrisiko haben. Gleichzeitig ergab sich in Beobachtungsstudien bei Frauen, die mit Metformin behandelt wurden, eine geringere Krebssterblichkeit. In einigen Studien konnte dies auch im Zusammenhang mit dem Mammakarzinom gezeigt werden. Um die Zusammenhänge näher zu untersuchen, schlossen US-Wissenschaftler 68.019 postmenopausale, brustgesunde Frauen zwischen 50 und 79 Jahren in eine prospektive Studie ein. 3.401 waren bereits zu Studienbeginn Diabetikerinnen, am Studienende waren es 11.290, die meisten davon übergewichtig oder adipös. 20,3\% der zuckerkranken Frauen nahmen zu Beginn der Studie Metformin als Monotherapie oder in Kombination ein, zum Studienende wurden 55\% mit Metformin behandelt. Während der Beobachtungszeit von knapp zwölf Jahren wurde bei 3.273 Frauen im Rahmen jährlicher Mammografien ein invasives Mammakarzinom und bei 754 Frauen ein duktales Karzinom in situ (DCIS) entdeckt. Wurde allein die Inzidenz von Brustkrebs bei Frauen mit und ohne Diabetes betrachtet, unabhängig davon, wie der Diabetes behandelt wurde, ergaben sich keine Unterschiede. Unter Berücksichtigung der Therapieform zeichnete sich bei Frauen unter Metformin gegenüber Nichtdiabetikerinnen allerdings ein niedrigeres Brustkrebsrisiko ab (Hazard Ratio [HR] 0,75). Dieser Zusammenhang ergab sich sowohl für östrogen- und progesteronabhängige Mammakarzinome als auch für HER2-negative Tumoren und blieb auch nach Berücksichtigung relevanter Risikofaktoren konstant. Bei Diabetikerinnen, die mit anderen Antidiabetika oder Insulin behandelt wurden, war das Brustkrebsrisiko leicht erhöht (HR 1,16).

Fazit: Auch in früheren Studien waren bereits positive Effekte von Metformin auf den Brustkrebs gesehen worden: So verlangsamte sich bei den Frauen z. B. die
Tumorproliferation, sie sprachen besser auf neoadjuvante Therapien an und hatten zum Teil einen günstigeren Verlauf. Durch welchen Mechanismus Metformin den Brustkrebs hemmt, ist noch unklar.
In weiteren Studie sollte der Einfluss auf verschiedene Brustkrebstypen gezielt untersucht werden, so die Autoren. Auch könne dann geklärt werden, inwieweit Metformin in der Prävention von Brustkrebs sowie im Therapiemanagement einsetzbar sei.

Christine Starostzik

Chlebowski RT et al. Diabetes, metformin, and breast cancer in postmenopausal women.

J Clin Oncol 2012;30(23):2844-52.

\section{Seltener Hormonersatztherapie, weniger Brustkrebs?}

In einigen Ländern war seit 2002 eine Abnahme der Brustkrebszahlen zu beobachten. Ob der zunehmende Verzicht auf eine Hormonersatztherapie dazu beitrug, wurde nun untersucht.

\begin{abstract}
— ine Hormonersatztherapie (HET)
— steigert das Brustkrebsrisiko, daher wurde ihre seltenere Anwendung infolge der Veröffentlichung der Studienergebnisse der Women's Health Initiative als

In Norwegen überlappen Effekte von HET-Reduktion und Einführung des Mammographie-Screenings auf die Brustkrebsinzidenz.
\end{abstract} Hauptgrund für die sinkende Brustkrebsinzidenz angesehen. Ob tatsächlich ein solcher Zusammenhang nachweisbar ist, wurde nun in Norwegen anhand der Daten aus dem norwegischen Krebsregister und der Verkaufszahlen für Hormonersatzpräparate untersucht.

Die Brustkrebsinzidenz stieg von 1956 bis zum Beginn des 21. Jahrhunderts in allen Altersgruppen an. Lediglich bei Frauen zwischen 25 und 39 Jahren blieb die Rate konstant. Besonders stark ausgeprägt war der Anstieg in den Jahren 1996 bis 2002 bei Frauen im Alter von 55 bis 69 Jahren, die in Bezirken wohnten, in denen das norwegische MammografieScreeningprogramm (Norwegian Breast Cancer Screening Program, NBCSP) als erstes eingeführt wurde. In Bezirken ohne Mammografie-Screening änderte sich die Brustkrebshäufigkeit dagegen nicht. Nach 2002/2003 sanken die Inzidenzraten wieder und blieben danach relativ konstant.

Bei Frauen im Alter von 55-59 Jahren war die Rate der hormonrezeptorpositiven Mammakarzinome in den Jahren 2000 bis 2003 am höchsten und fiel dann wieder $\mathrm{ab}$. In anderen Altersgruppen wurde kein eindeutiger Peak beobachtet. Bei 50 - bis 54 -jährigen und bei 60 - bis 64 -jäh-

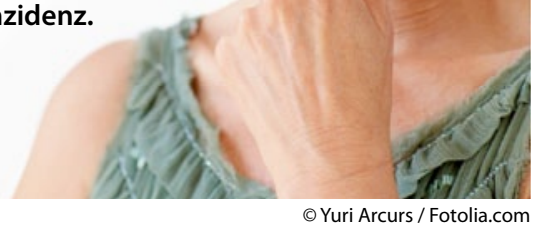

rigen Frauen stieg die Inzidenz der hormonrezeptorpositiven Mammakarzinome während der gesamten Beobachtungsdauer an. Bei älteren Frauen ergab sich eine U-förmige Kurve mit den niedrigsten Zahlen in den Jahren 1999 bis 2005.

Die Verkaufszahlen der Hormonersatzpräparate stiegen von 1987 bis 2001 an und sanken danach wieder.

Fazit: Die Entwicklung der Brustkrebsinzidenz in Norwegen von 1987 bis 2009 ist schwierig $\mathrm{zu}$ interpretieren, weil das Mammografie-Screeningprogramm zu der Zeit eingeführt wurde, als die HET zunehmend häufiger zum Einsatz kam. Beide Faktoren beeinflussen die beobachteten Trends. Ihre Bedeutung ist vermutlich in verschiedenen Altersgruppen unterschiedlich.

Judith Neumaier

Hofvind S et al. Breast cancer incidence trends in Norway - explained by hormone therapy or mammographic screening? Int J Cancer. 2012;130(12):2930-8. 